\title{
Study of the mechanisms underlying the fresh-state response of cementitious materials modified with nanoclays
}

\author{
Shiho Kawashima*, Jae Hong Kim, David J. Corr, Surendra P. Shah \\ Northwestern University, Civil and Environmental Engineering, 2145 Sheridan Road, Evanston, IL 60208, USA
}

\section{H I G H L I G H T S}

- Nanoclays have an immediate stiffening effect on fresh cement paste, little over time.

- Stiffening due to flocculation, not adsorption by nanoclays leading to loss of free water.

- Clay effect on effective solid volume fraction, $\phi_{f}$, and maximum packing density, $\phi_{M}$.

- Describe paste flow properties and SCC formwork pressure response through $\phi_{f}$ and $\phi_{M}$.

\section{A R T I C L E I N F O}

\section{Article history:}

Received 20 April 2011

Received in revised form 2 May 2012

Accepted 4 June 2012

Available online 15 July 2012

\section{Keywords:}

Concrete

Cement paste

Nanoclay

Rheology

Fresh-state

Formwork pressure

Flocculation

\begin{abstract}
A B S T R A C T
The fresh-state properties of concrete can be tailored to meet the needs of specific applications, such as reduced formwork pressure for self-consolidating concrete (SCC), which requires high flowability, and slipform paving concrete, which requires compactibility during casting followed by enhanced green strength immediately after placement. Small additions of clays (especially nanoclays) have been found to be very effective in achieving these properties. The purpose of this study was to examine concentrated cement-clay systems at multiple scales to gain a better understanding of the mechanisms underlying this behavior. This was done by investigating the macroscopic flow properties over time through a shear rheological approach, where the viscosity evolution under a constant applied shear rate and a measure of structural rebuilding over time were obtained. The measured flow parameters were used to describe the microstructure in the plastic state through the Krieger-Dougherty model. This was then tied in with results obtained previously through scanning laser microscopy, a direct measure of the microstructure by focus beam reflectance method (FBRM). The dominating mechanisms behind the stiffening behavior were determined, and ultimately used to explain the response of SCC for lab-scale simulations of formwork pressure. The effective solid volume fraction and maximum packing density were found to be useful parameters in doing so, and helped to explain why nanoclays are an effective mineral admixture in modifying SCC for reducing formwork pressure.
\end{abstract}

(c) 2012 Elsevier Ltd. All rights reserved.

\section{Introduction}

The continued advancement of self-consolidating concrete (SCC) hinges on a firm understanding of its fresh-state properties. Through innovative processing, conventional SCC can be designed to achieve high flowability, passing ability, and stability, all of which enhance construction productivity. The fresh-state properties of SCC can be further tailored to meet the needs of specific applications, such as reduced formwork pressure and slipform paving concrete, which require compactibility during casting followed by enhanced green strength (strength of freshly-cast concrete in

* Corresponding author. Address: 2145 Sheridan Road, Suite A130, Evanston, IL 60208, USA. Tel.: +1 $8474917161(0)$.

E-mail address: s-kawashima@northwestern.edu (S. Kawashima). the plastic state) immediately after placement. Small additions of clays (especially nanoclays) have been found to be very effective in achieving these properties. Although this has been linked to increases in fresh-state stiffness (resistance to flow) caused by the clays, no direct correlations have been made so the mechanisms are still not well understood. This requires examining the clay-cement system at multiple scales.

Previous studies relating to this topic have provided insight into how clays affect the microstructural properties of fresh cement pastes. Results of shear and compressive rheology and scanning laser microscopy tests have shown that clays increase flocculation strength and floc size [1,2]. Although this explains the apparent increase in stiffness caused by the clays, it is not sufficient in explaining how it translates to the reduction in SCC formwork pressure observed in lab-scale tests. It is necessary to provide a link 
between the microstructural properties and the response of the materials designed for this application. The purpose of this study was to do so by investigating the macroscopic flow behavior over time of cement pastes with and without small nanoclay additions. This was done through a shear rheological approach, where the viscosity evolution under a constant applied shear rate and a measure of structural rebuilding over time were obtained. The measured flow parameters were used to describe the microstructure in the plastic state through the Krieger-Dougherty model. This was then tied in with results obtained previously through scanning laser microscopy, a direct measure of the microstructure by focus beam reflectance method (FBRM). The dominating mechanisms behind the stiffening behavior were determined, and ultimately used to explain the response of modified SCC mixes designed for reduced formwork pressure during casting.

\section{Background}

Very small additions of clays (up to $1.5 \%$ by mass of cement) lead to significant changes in the fresh-state properties of cement-based materials, which may be tailored to meet the needs of specific applications. A study on extruded cement-based materials demonstrated that cellulose ethers can be partially replaced by two types of kaolinite clays [3]. At additions as low as $0.15 \%$ by mass, mixes were able to flow through the die at sufficiently low pressures and exhibit shape stability upon exiting. In studies that simulated slipform (SF) paving with a lab-scale minipaver, various types of clays were found to be an integral mineral admixture in low compacting energy concrete, SF-SCC, mix design [4-7]. SF paving is widely used for concrete pavement construction, where placing, consolidating, and finishing of fresh concrete are done in one continuous process. SF-SCC makes it possible to eliminate external vibration from this process. Not only does this make it less energy intensive but it helps to avoid problems associated with over-consolidation, such as segregation and significant reduction of entrained air, which lead to reduction in durability and extensive longitudinal cracking along the path of the vibrators. SF-SCC containing fly ash and superplasticizers exhibited high fluidity and smooth surface finish but poor shape stability. A significant improvement was observed with small additions of clays, where straight edges were achieved after placement with little compromise to initial flowability and compactibility.

The motivation behind the present study was to better understand the response of clay-modified SCC designed for the application of reducing formwork pressure. Although the use of SCC is becoming more prevalent in pre-cast plants, there is still apprehension when extending it to cast-in-place applications. This is partially attributed to the high lateral pressure SCC exerts on formwork from increased fluidity and faster casting rates compared to vibrated concrete. There was a study that investigated the effect of various mineral admixtures on SCC formwork pressure response [8], where the formwork pressure evolution of SCC concrete mixes were measured using a formwork pressure device (depth, $D=15 \mathrm{~cm}$ and height, $H=30 \mathrm{~cm}$ ), shown in Fig. 1 . This setup simulated the lateral pressure induced on formwork during casting, allowing for different casting speeds and heights. Vertical pressure is applied directly to the surface of the concrete using a steel piston loaded by a closed-loop hydraulic testing frame and the lateral pressure is recorded by a transducer. (The details of the test setup can be found elsewhere [8].) The results for SCC with $0 \%$ (NCO) and $0.33 \%$ (NC0.33) nanoclays are shown in Fig. 2. Although both mixes exhibited the same initial flowability with a slump flow of $60 \pm 2 \mathrm{~cm}$ (which fulfills the requirement for SCC), it is apparent that even at small dosages clays can lead to significantly reduced

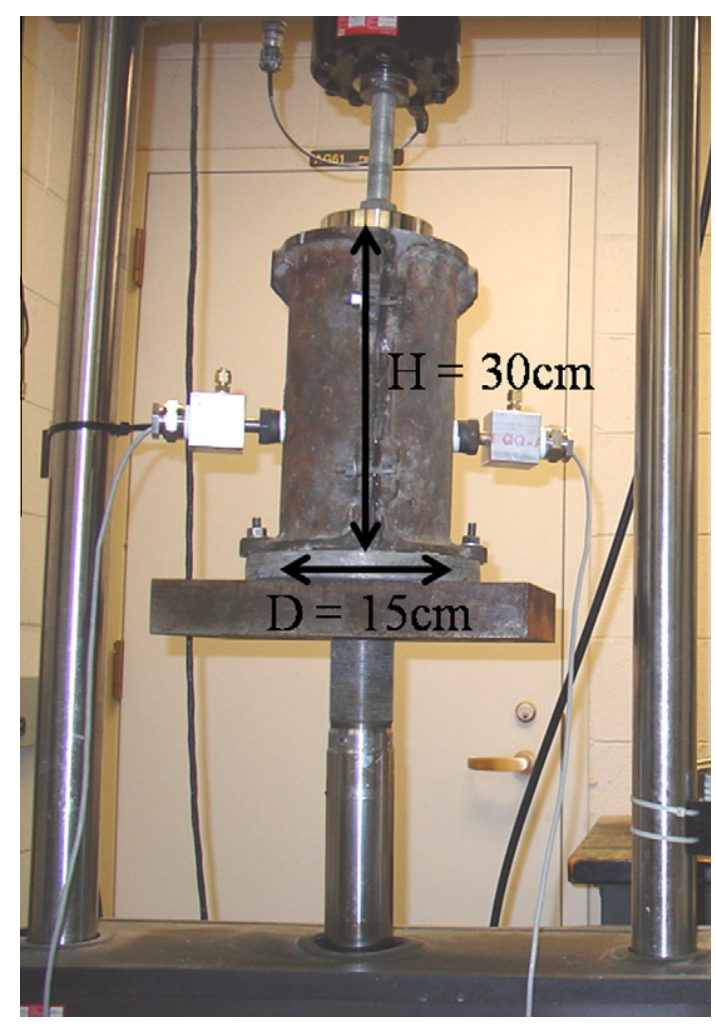

Fig. 1. Formwork pressure device with a depth $D=15 \mathrm{~cm}$ and height $H=30 \mathrm{~cm}$.

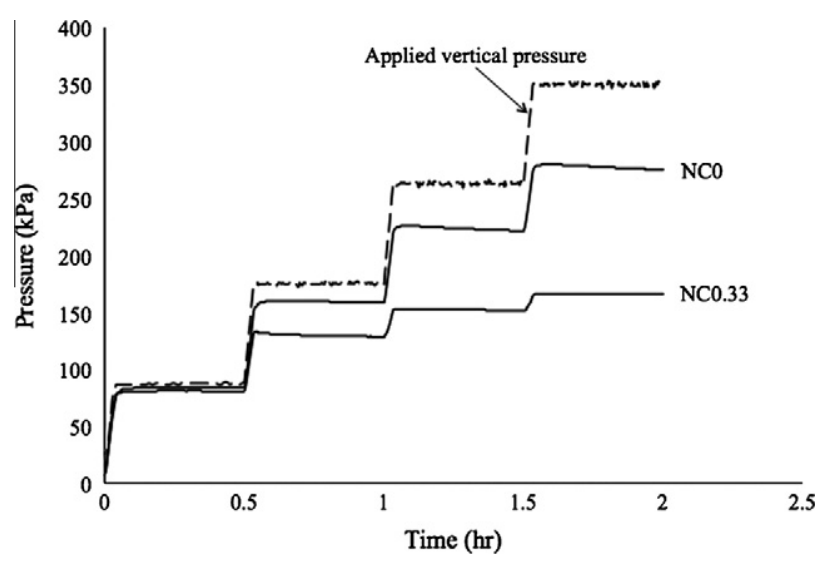

Fig. 2. Formwork pressure response of SCC with and without nanoclays (both mixes had an initial slump flow of $60 \pm 2 \mathrm{~cm}$ ) [8].

formwork pressure. However, to explore the mechanisms behind this behavior was not within the scope of that study.

To most effectively tailor fresh-state properties for application through the use of nanoclays, it is necessary to understand the mechanisms underlying the stiffening behavior. Possible mechanisms include capillary suction [9], hydration mechanisms, water adsorption, particle packing, and flocculation. However, hydration can be considered to be negligible due to the time scale of the observed behavior (within $2 \mathrm{~h}$ after initial cement-water contact). The contribution of dry clay on particle packing is unlikely a significant factor due to the small dosage (less than $1 \%$ by mass of cement). And capillary suction is thought to be an effect of flocculation. This leaves flocculation and water adsorption, both of which can be studied through flow parameters and the Krieger-Dougherty equation, which will be explained in the 
proceeding section. Previous studies have demonstrated that clays increase flocculation strength and floc size. As for water adsorption, if the water content is kept constant, the inclusion of any nanoparticles (due to their high specific surface area) would reduce free water and result in increased yield stress and viscosity [10]. Therefore it is important to evaluate whether water adsorption by the nanoclays could be a dominating mechanism.

SCC formwork pressure has been linked to degree of thixotropy (a decrease in viscosity under shear followed by a recovery once the stress is removed [11]), where increased thixotropy leads to decreased formwork pressure [12,13]. Although thixotropy likely plays a role and the influence of clays on this property deserves attention, this present study focused on the overall stiffening behavior, without separating reversible and irreversible changes.

\section{Theoretical background for data analysis}

\subsection{Krieger-Dougherty equation}

The Krieger-Dougherty model [14] describes the relationship between viscosity and solid volume fraction for dispersed suspensions:

$\frac{\eta}{\eta_{c}}=\left(1-\frac{\phi}{\phi_{M}}\right)^{-[\eta] \phi_{M}}$

where $\eta$ is the apparent viscosity of the suspension, $\eta_{c}$ is the apparent viscosity of the continuous phase (or liquid phase), $\phi$ is the concentration of solids by volume, $[\eta]$ is the intrinsic viscosity of the suspension, and $\phi_{M}$ is the maximum solids concentration by volume, or maximum packing density. $\phi_{M}$ depends on particle size distribution and shape, while $[\eta]$ depends on particle shape - both are shear dependent. Derivations of the model can be found elsewhere $[15,16]$. It is apparent from the equation that an increase in $\phi$ would lead to higher viscosity, assuming $\phi_{M}$ and $[\eta]$ remain constant.

Cement pastes are highly concentrated, highly flocculated suspensions. Flocculation leads to entrapped water within particles, resulting in a change in the solid volume fraction. To take this into consideration, Soua et al. [17] proposed the following modified model, which is an extension of the Krieger-Dougherty equation for flocculated suspensions:

$\frac{\eta}{\eta_{c}}=\left[b\left(1-\frac{\phi_{f}}{\phi_{f M}}\right)\right]^{-2} \quad$ for $\quad \tau>\tau_{y}$

where $\phi_{f}$ is the effective volume fraction (floc volume fraction), $\phi_{f M}$ is the maximum packing density of the flocs, $b$ is an adjusting factor, $\tau$ is applied shear stress, and $\tau_{y}$ is yield stress. And it follows that at sufficiently high shear $\phi_{f} \rightarrow \phi$ and $\phi_{f M} \rightarrow \phi_{M}$ as the flocs continue to break down and release entrapped water.

The flow behavior of cement paste was found to be well-described by the Krieger-Dougherty model [18,19], although the parameters may not have the same physical significance as they do for non-flocculated systems. For cement paste, the maximum packing density is of great interest because it can provide a measure of the degree of flocculation in the system, with higher degree of flocculation leading to lower $\phi_{M}$. Tregger et al. used the modified model to evaluate the influence of clays on flocculation strength, the resistance of flocs to break down under a given shear condition $[1,20]$. Results showed that cement paste with small clay additions yielded lower $\phi_{M}$ values compared to neat cement paste, indicating that clays lead to a more flocculated structure by increasing flocculation strength. This study was limited to obtaining the properties of the paste shortly after mixing. One of the main objectives of the present work is to see how the clays are affecting $\phi_{M}$, as well as $\phi$, over time.

The evolution of packing density is mainly due to flocculation or agglomeration, the process of which may be simply stated as the transition from single cement particles into flocs. The well-known theory of packing density of non-crowding binary particles shows the relationship between the fractional solid volume of one component and the maximum packing density of the entire system. The details of the theory can be found elsewhere [21] and will briefly be explained here. In a binary system, there are two components (small grains and large grains) where each component $i$ has a packing density, $\alpha_{i}$. The fractional solid volume of each component, $\left[\phi_{i}\right]$, is defined as:

$\left[\phi_{i}\right]=\frac{\phi_{i}}{\phi_{\mathrm{M}}}$

where $\phi_{i}$ is the partial volume of component $i$ and $\phi_{M}$ is the packing density of the binary system. There are two possible conditions. The first is where the large grains are fully packed and the remaining interstitial space is composed of the small grains. Following the convention $r_{1}>r_{2}$, the packing density for this condition can be represented by the following expression:

$\phi_{M, 1}=\frac{\alpha_{1}}{1-\left[\phi_{2}\right]}$

As $\left[\phi_{2}\right]$ increases (volume of small grains increases), the packing density of the system increases. The second condition is when the small grains are fully packed:

$\phi_{M, 2}=\frac{\alpha_{2}}{1-\left(1-\alpha_{2}\right)\left[\phi_{1}\right]}$

Here, it is apparent that as $\left[\phi_{1}\right]$ increases (volume of large grains increases), $\phi_{M}$ increases. Fig. 3 shows the packing density of the system as a function of $\left[\phi_{2}\right]$.

Extending this model to a cement-water system, the two components can be considered to be single cement particles $\left(r_{2}\right)$ and flocs $\left(r_{1}\right): r_{2}<r_{1}$. It follows that in the highly flocculated state cement paste will follow the $\phi_{M, 1}$ curve whereas in the highly dispersed state it will follow the $\phi_{M, 2}$ curve. Considering that single cement particles follow a particle size distribution (PSD) and flocs vary in size, this is a simplified assumption. Still, the model can be used to extract useful qualitative information about $\phi_{M}$ from the results of FRBM tests. FRBM is a particle size analysis measurement technique that provides information about particle chord length distribution in real time [22]. It is a direct measure of the microstructure of cement paste in its plastic state, whereas in

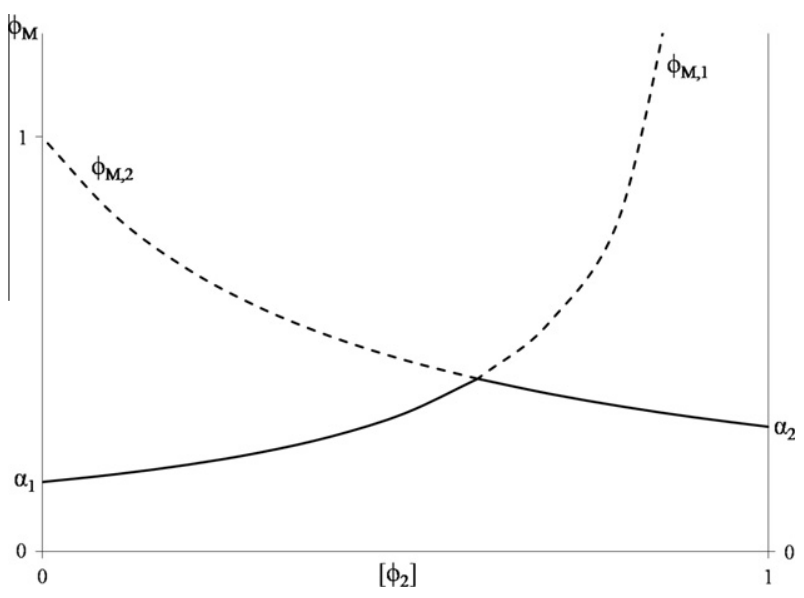

Fig. 3. Packing density of a binary system, $\phi_{M}$, as a function of [ $\left.\phi_{2}\right]$. 
shear rheological methods it is described indirectly using the Krieger-Dougherty model. In a previous study FBRM tests were conducted on cement pastes with and without clays subjected to shear and the evolution of average floc size were compared [2].

\section{Experimental methods and materials}

\subsection{Materials}

Type I ordinary Portland cement and tap water were used in all paste mixes. A purified magnesium aluminosilicate, or palygorskite, was selected for this study, as it exhibited the most enhanced effect in previous studies compared to other clays. For example, in the flocculation study by Tregger et al., this clay yielded the lowest $\phi_{M}$ value, indicating that it led to the greatest increase in flocculation strength. They are nanoclays that have been chemically exfoliated to preserve their uniform shape and size while removing all impurities (such as quartz and swelling clays). As received, they are highly agglomerated - the scanning electron microscopy (SEM) image, Fig. 4a shows that the agglomerates are on the micron scale. However, in the highly dispersed state they are nanosized particles with a needle-like shape (1.75 $\mu \mathrm{m}$ in length and $3 \mathrm{~nm}$ in diameter) [23], Fig. 4b.

\subsubsection{Water adsorption of nanoclays}

In the dispersed state, the high specific surface area of the nanoclays in combination with their small particle size can result in a significantly high adsorption capacity. The water adsorption of the nanoclay was measured through a pressure filtration setup used in similar work on alite $\left(C_{3} S\right)$ [19], shown in Fig. 5. A sample of dry nanoclay was placed on a fine filter $(0.45 \mu \mathrm{m}$ membrane $)$ over which a layer of water was carefully poured so as not to disturb it. A constant negative pressure was applied at the bottom by a vacuum, which pulled the water through the nanoclays to form a cake and consolidate it. Water that was not retained was filtered out into an Erlenmeyer flask. The water over the cake was kept at a constant level and the pressure was applied until a constant flow rate was achieved (after
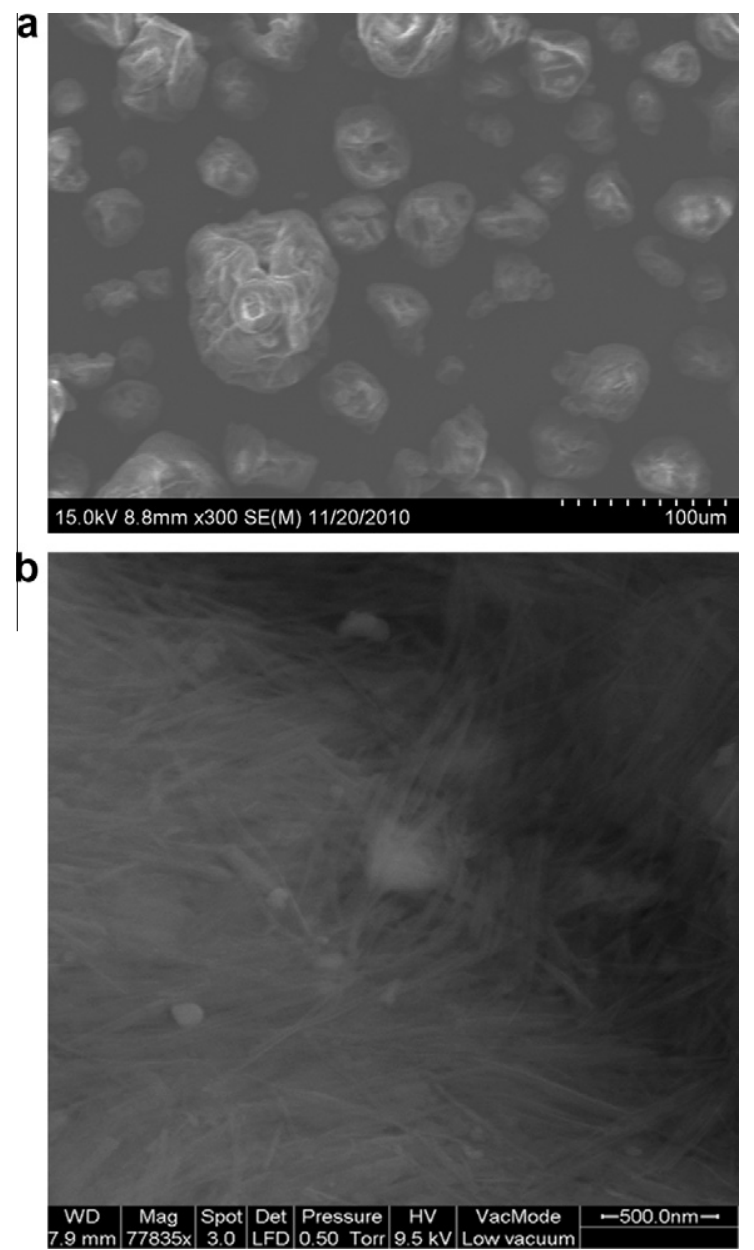

Fig. 4. SEM image of nanoclay in the (a) agglomerated state and (b) dispersed state [31].

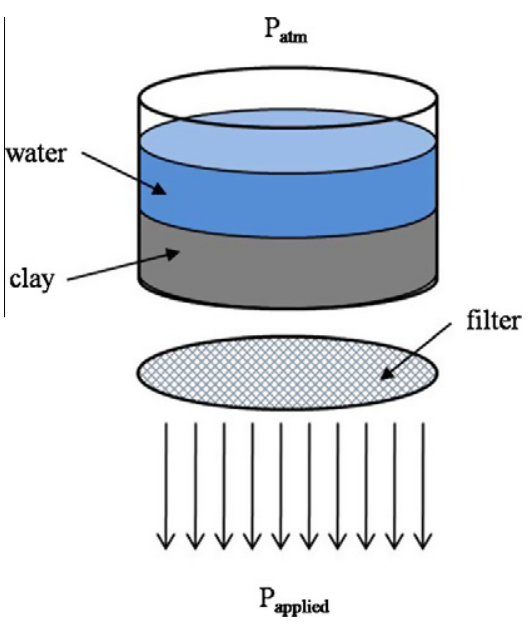

Fig. 5. Schematic of filtration setup to measure water adsorption of nanoclay.

approximately $90 \mathrm{~min}$ ) at which point the cake reached a state of equilibrium (full adsorption of the nanoclays and full saturation of the cake). At sufficiently high pressures, it is assumed that the cake is consolidated enough to contain only the nanoclays and the adsorbed water. A portion of the cake was then carefully removed, the wet mass ( $m_{\text {wet }}$ ) was recorded and then oven dried at $105{ }^{\circ} \mathrm{C}$ for $3 \mathrm{~h}$ to evaporate off all of the water. The sample was placed in a desiccator for 30 min before the dry mass ( $m_{\mathrm{dry}}$ ) was recorded. From the wet and dry mass, the water adsorption of the clays was calculated:

Adsorption $(\%)=\frac{m_{\text {wet }}-m_{\text {dry }}}{m_{\text {dry }}} \times 100 \%$

This was performed at increasing applied pressures until the adsorption values converged. The average of three measurements was taken to be the representative water adsorption at each applied pressure. The results are shown in Fig. 6. At lower pressures, the cake was not fully consolidated and contained excess water. At higher pressures, the values converged to approximately $200 \%$ by dry mass of nanoclay, which agrees with reported values by the manufacturer [23] and other literature [24].

\subsection{Dispersion}

The nanoclays used in this study were hydrophilic, which made them easily dispersible in water. To determine the influence of dispersion on fluidity, the minislump flow of cement pastes were measured and compared for different mixing methods. Pastes were prepared either by a small planetary mixer or a household blender for high shear mixing. The mixing protocols are shown in Table 1 . In the case of the planetary mixer, the nanoclays were either undispersed and added asreceived in the dry, agglomerated form (P1) or pre-dispersed in water at high shear in a blender prior to mixing (P2). The mini-slump flow tests were performed immediately after mixing based on ASTM C 1611 [25], where a Hägermann cone

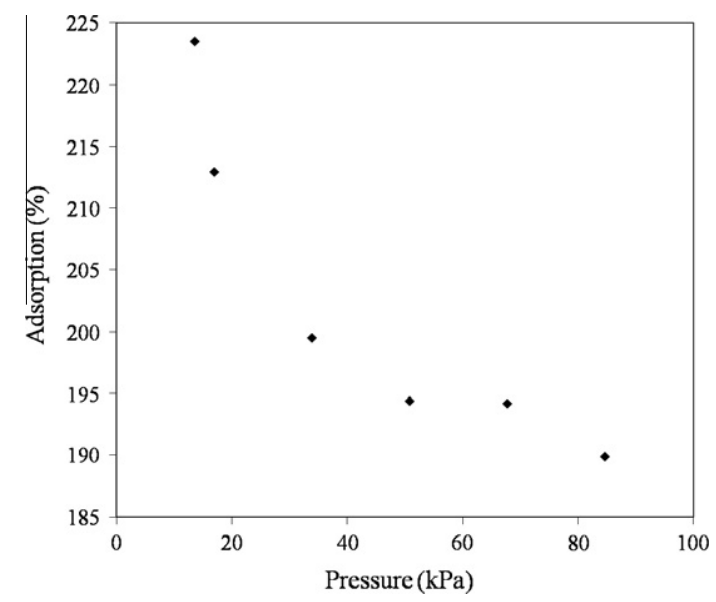

Fig. 6. Water adsorption of nanoclays at various applied pressures as measured in the filtration test. 
Table 1

Mixing protocols.

\begin{tabular}{|c|c|c|}
\hline Protocol & Mixer & Mixing procedure \\
\hline P1 & Planetary & $\begin{array}{l}\text { (1) Add cement (and nanoclay) to bowl. Mix for } 2 \text { min at speed } 1 \\
\text { (2) Add water, mix for } 1 \text { min at speed } 1 \text { scrape bowl, mix for } 2 \text { min at speed } 1 \text { scrape bowl, mix for } 4 \text { min at speed } 2\end{array}$ \\
\hline P2 & Planetary & $\begin{array}{l}\text { (1) Add nanoclay and water to blender. Blend for } 2 \text { min } \\
\text { (2) Add nanoclay-water suspension to dry cement in bowl } \\
\text { (3) Step } 2 \text { of protocol P1 }\end{array}$ \\
\hline B & Blender & $\begin{array}{l}\text { (1) Add water (and nanoclay) to blender. Blend for } 1 \mathrm{~min} \\
\text { (2) Add half of cement. Blend for } 15 \mathrm{~s} \\
\text { (3) Add rest of cement, blend for } 15 \mathrm{~s} \text {, scrape. Blend until total mixing time is } 3 \mathrm{~min}\end{array}$ \\
\hline
\end{tabular}

with $100 \mathrm{~mm}$ lower diameter, $70 \mathrm{~mm}$ upper diameter and $50 \mathrm{~mm}$ height was used. One slump flow measurement was taken for each mix. All paste mixes had a volume fraction of 0.43 and a nanoclay addition of $0 \%$ or $0.5 \%$ by mass of cement.

\subsection{Viscosity evolution}

A shear rheological method was used to obtain the viscosity evolution of cement pastes under a constant shear condition, which will be referred to as the constant rate $(\mathrm{CR})$ protocol herein. All shear rheology tests were performed in a temperature-controlled rheometer with a coaxial cylinder geometry set at room temperature. The CR protocol is shown in Fig. 7. A constant shear rate of $300 \mathrm{~s}^{-1}$ was applied to paste samples for $60 \mathrm{~min}$, from which the viscosity evolution was obtained.

The mix compositions tested are shown in Table 2. A plain cement paste mix (NCO) and a mix with $1 \%$ addition of nanoclays by mass of cement (NC1) were tested, both with a volume fraction of 0.43 . In addition, each mix was adjusted for water adsorption of the clays. $\mathrm{NC} 1 \mathrm{H}$ is $\mathrm{NC} 1$ with an additional $10 \mathrm{~g}$ of water to compensate for the amount adsorbed by the $5 \mathrm{~g}$ of nanoclay while $\mathrm{NCOH}$ is NC0 with $10 \mathrm{~g}$ less water. (200\% water adsorption by mass of clay was assumed according to the results of the filtration test.) For the $\mathrm{CR}$ test, pastes were prepared by following protocol B in Table 1, subjected to high shear mixing so that they could be placed in the rheometer as quickly as possible after initial cement and water contact. This was considered to be important because the adsorption rate of the nanoclays was unknown. This test was performed to determine whether water adsorption leading to a loss of free water was a dominating factor in the increase in stiffness caused by the clays. Three measurements were taken for each mix.

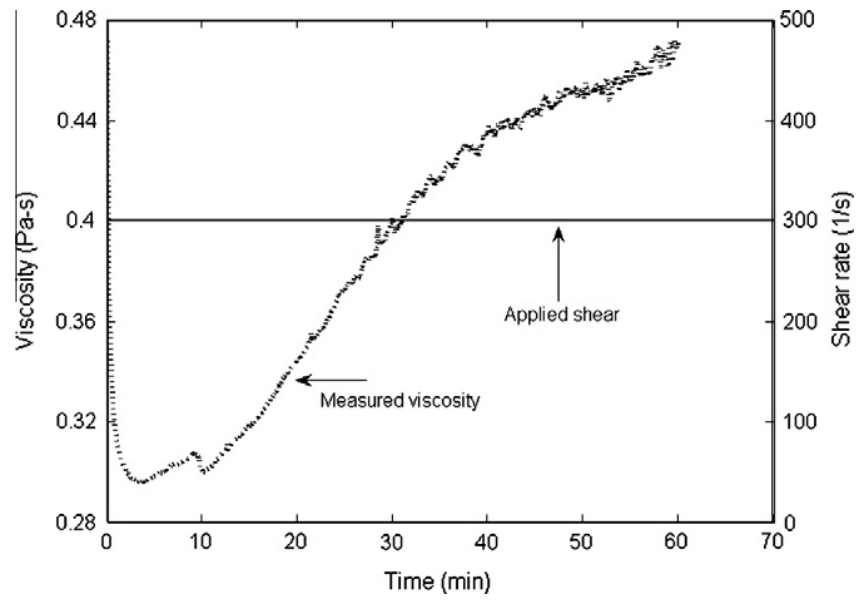

Fig. 7. Applied shear rate and measured viscosity plotted against time for $C R$ protocol.

Table 2

Mix proportions for constant applied shear rate protocol.

\begin{tabular}{lllll}
\hline Mix & Cement $(\mathrm{g})$ & Water $(\mathrm{g})$ & Clay $(\mathrm{g})$ & Solid volume fraction \\
\hline NCO & 500 & 215 & 0 & 0.43 \\
NC1 & 500 & 217.6 & 5 & 0.43 \\
NC1H & 500 & 227.6 & 5 & 0.41 \\
NCOH & 500 & 205 & 0 & 0.44 \\
\hline
\end{tabular}

\subsection{Specific rebuilding energy (SRE)}

In the $\mathrm{CR}$ protocol, the material was subjected to a constant high shear. To investigate the stiffening behavior when it is left at rest, the specific rebuilding energy (SRE) protocol, developed by Ferron et al., was applied [26].

The applied shear rate for the SRE protocol is shown in Fig. 8. The paste sample was subjected to a pre-shear at a high rate $\left(600 \mathrm{~s}^{-1}\right)$ for $10 \mathrm{~min}$ to break down its inherent microstructure until an equilibrium state was reached. Then the shear was linearly ramped down to $0 \mathrm{~s}^{-1}$ over $30 \mathrm{~s}$ to obtain an equilibrium curve. The mix was at rest for the remainder of the test, with the exception of a hysteresis loop (linearly ramped up to $300 \mathrm{~s}^{-1}$ and back down to $0 \mathrm{~s}^{-1}$ over $30 \mathrm{~s}$ ) applied every $15 \mathrm{~min}$ up to $60 \mathrm{~min}$. The area between each loading curve and the equilibrium curve between $100 \mathrm{~s}^{-1}$ and $200 \mathrm{~s}^{-1}$ is defined as the SRE, as shown in Fig. 9. Also given in the following expression:

$\mathrm{SRE}=\int_{\dot{\gamma}=100}^{\dot{\gamma}=200}\left(\tau_{\text {Loading }}-\tau_{\text {Equil }}\right) d \dot{\gamma}$

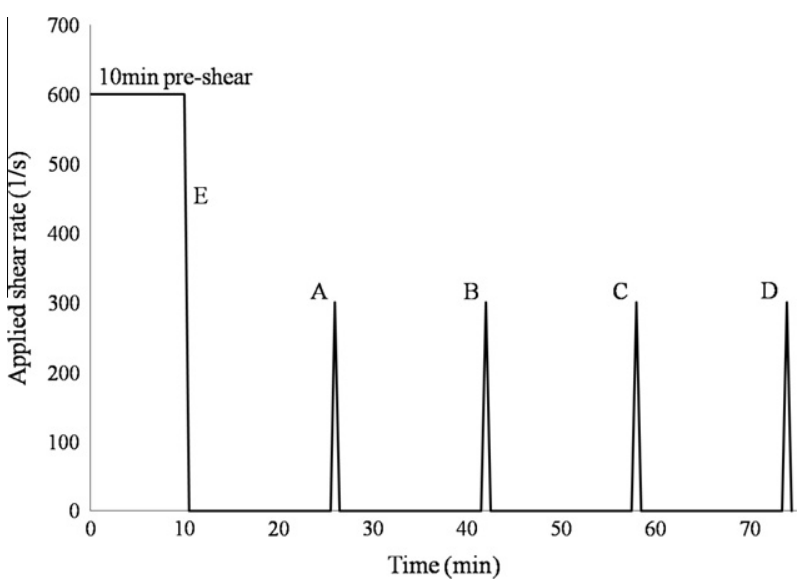

Fig. 8. Applied shear rate for SRE protocol.

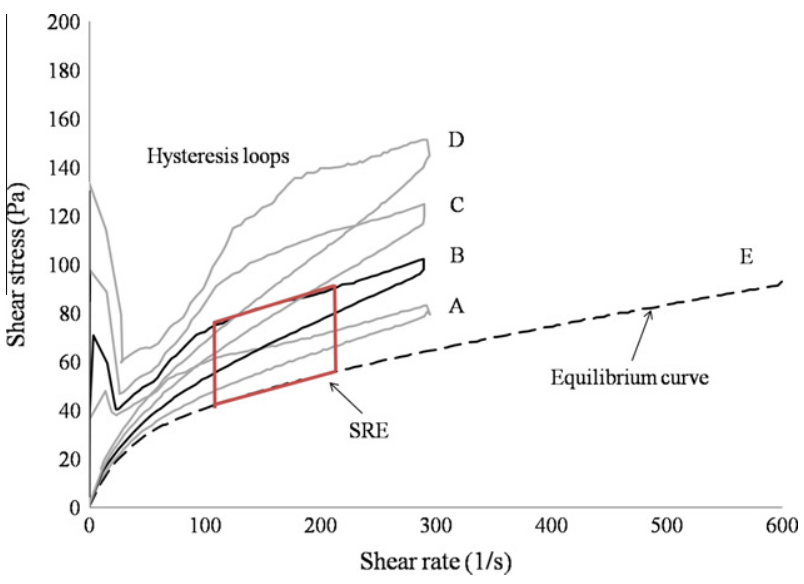

Fig. 9. Shear stress versus shear rate curves obtained from SRE protocol. 
where $\tau$ is shear stress and $\dot{\gamma}$ is strain rate. The rate at which SRE increases provides a measure of structural rebuilding of the material over time. Cement pastes with (NC0.3) and without (NCO) a $0.3 \%$ nanoclay addition by mass of cement were tested. For the SRE test protocol P2 was followed. Three measurements were taken for each mix.

In addition, the flow curve parameters, yield stress and viscosity, were obtained from the equilibrium curve to compare the initial state of the samples. A modified Bingham equation was used [27]:

$\tau=\tau_{y}\left[1-\exp \left(3 \cdot \frac{\dot{\gamma}}{\dot{\gamma}_{\text {crit }}}\right)\right]+\mu \dot{\gamma}$

where $\tau_{y}$ is yield stress, $\dot{\gamma}_{\text {crit }}$ is critical strain rate corresponding to $\tau_{y}$, and $\mu$ is plastic viscosity.

\section{Experimental results}

\subsection{Dispersion}

To determine the influence of dispersion, the slump flow of cement pastes were measured and compared for various mixing methods. The results are shown in Table 3. As expected, the plain cement paste (NCO) experienced higher fluidity when mixed in a blender than in a planetary mixer, due to a higher degree of deflocculation. On the other hand, in the case of the cement paste with $0.5 \%$ addition of clay (NC0.5), the opposite was observed - the mix prepared in the blender was much stiffer. In addition, the mix also experienced a significant decrease in fluidity when the clays were pre-dispersed in water with a blender prior to mixing in a planetary mixer with the cement. The results illustrate that high degree of dispersion of the clays maximize their effect, likely due to increased surface interactions between the nanoclays and the cement particles. Also, the stiffening effect of the clays occurs immediately after placement.

\subsection{Viscosity evolution}

Fig. 10 shows the results of the CR protocol. Fig. 10a is the viscosity evolution up to $5 \mathrm{~min}$, during which each mix reached equilibrium. In evaluating the effect of adjusting for water adsorption of the clays, if loss of free water is a governing stiffening mechanism, mixes $\mathrm{NCO}$ and $\mathrm{NC} 1 \mathrm{H}$ and mixes $\mathrm{NC} 1$ and $\mathrm{NCOH}$ should have exhibited similar viscosity evolutions. The plot shows that the viscosity at equilibrium of these mixes did not coincide: $\eta_{\mathrm{NC1H}}>\eta_{\mathrm{NCO}}$ and $\eta_{\mathrm{NC} 1}>\eta_{\mathrm{NCOH}}$. Also, the curves follow different trends - both clay mixes show a significant decrease in viscosity while the neat cement pastes do not. It is apparent that adjusting the water content was not sufficient in negating the effect of the clays on stiffening, thereby making it unlikely that water adsorption leading to loss of free water is a dominating factor.

The behavior of the clay mixes can be explained using Eq. (2), which shows viscosity to be directly related to effective volume fraction. After the material is placed and at rest, $\phi_{f}$ increases instantaneously, indicated by the high viscosity measured at the start of the protocol upon introduction of shear. This also shows that the effect of the clays on stiffness occurs rapidly, within 5 min after initial cement-water contact. Viscosity decreases as

Table 3

Results of slump flow test to determine effect of nanoclay dispersion.

\begin{tabular}{lll}
\hline Mix & Protocol & Slump flow $(\mathrm{cm})$ \\
\hline NC0 & P1 & 18.2 \\
& B & 23.8 \\
NC0.5 & P1 & 18.3 \\
& P2 & 11 \\
& B & 11 \\
\hline
\end{tabular}
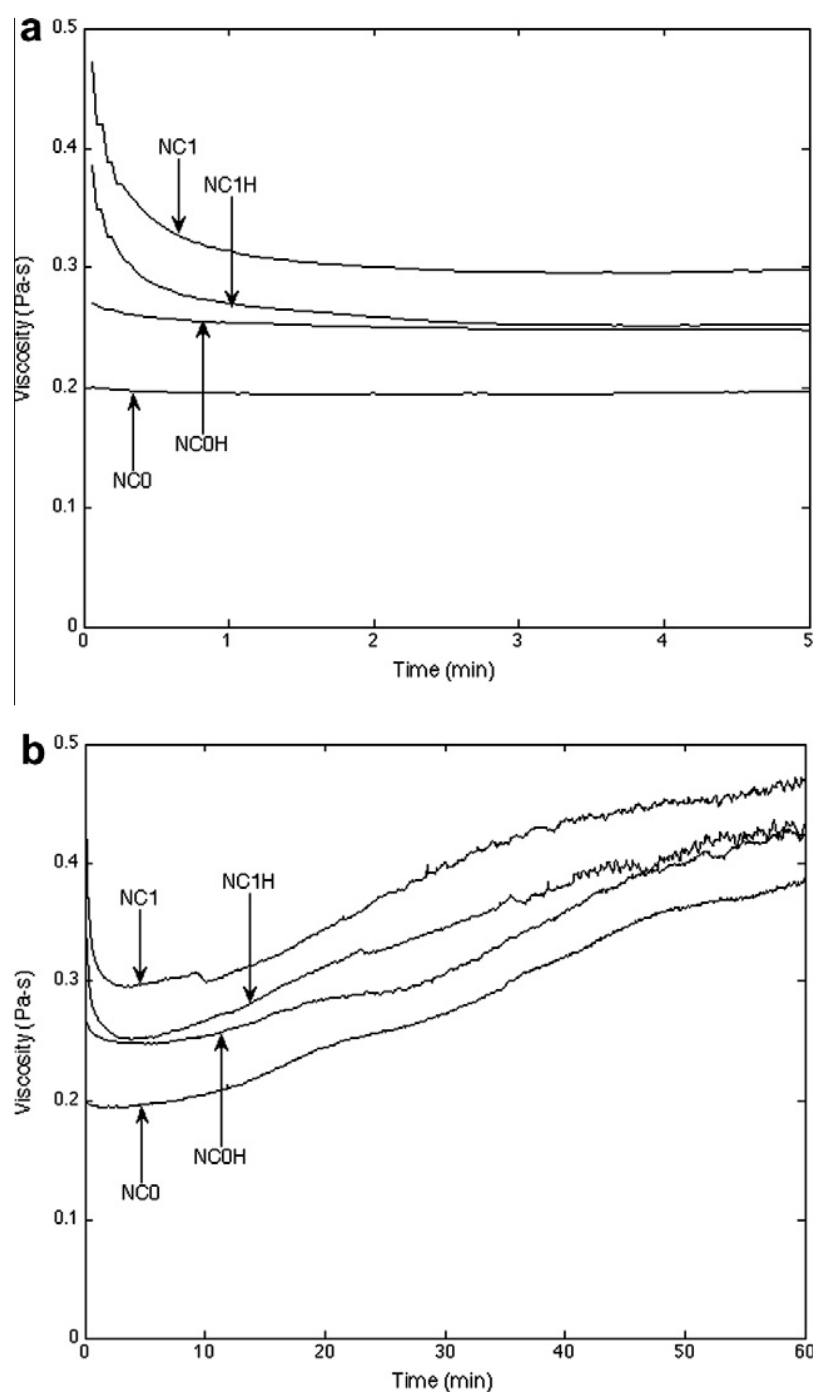

Fig. 10. Viscosity evolution (a) up to equilibrium ( $5 \mathrm{~min}$ ) and (b) after equilibrium (up to $60 \mathrm{~min}$ ).

the material continues to be sheared, indicating decreasing $\phi_{f}$. This is likely due to deflocculation, during which changes occur in the solid and water content. It follows that flocculation induced by the clays is the dominating mechanism underlying the stiffening behavior.

After equilibrium, at which point flocs cannot be broken down any further under the given shear condition, viscosity increased for all pastes, as shown in Fig. $10 \mathrm{~b}$. As expected, $\eta_{\mathrm{NC} 1}>\eta_{\mathrm{NCO}}$ at equilibrium due to the increase in flocculation strength induced by the clays $\left(\phi_{M, \mathrm{NC} 1}<\phi_{M, \mathrm{NCO}}\right)$. However, both mixes experienced a similar rate of stiffening over time. Table 4 shows the change in viscosity of all mixes from equilibrium to final ( $60 \mathrm{~min})$. The values are all very close, indicating that the stiffening over time under the constant shear condition was not influenced by the clays. Referring back to the Krieger-Dougherty equation, Eq. (1), changes in viscosity may be attributed to changes in the parameters $\phi$ and/or $\phi_{M}$. Increasing viscosity can result from increasing $\phi$, decreasing $\phi_{M}$, or a combination of both. The results of the CR protocol alone are not sufficient in determining which parameter is governing this behavior over time, but they do show that it is attributed to the properties of the cement particles (e.g. adsorption or flocculation behavior), not of the clays. 
Table 4

Change in viscosity from equilibrium to final (60 min) of pastes under constant shear.

\begin{tabular}{ll}
\hline Mix & $\Delta$ Viscosity (Pa-s) \\
\hline NCO & 0.192 \\
NC1 & 0.175 \\
NC1H & 0.18 \\
NCOH & 0.176 \\
\hline
\end{tabular}

\subsection{Structural rebuilding}

The results of the SRE protocol are shown in Figs. 11 and 12. It is apparent that the clay mix experienced greater yield stress and viscosity at equilibrium, as shown in Table 5 and Fig. 11. However, the two mixes went onto experience the same SRE rate, indicating similar structural rebuilding (Fig. 12). A change in the water or solids content would lead to a change in the SRE rate. For instance, mixes with higher water content would experience lower SRE rates due to a reduction in coagulation forces and interparticle collisions. Therefore, the results show that the clays did not have a significant effect on the water or solids content after equilibrium and the mechanism behind structural rebuilding must be linked to the cement particles. This agrees with the results of the constant applied shear rate protocol. The clays have an immediate effect on thixotropy, likely due to flocculation, but then have little influence over time.

\section{Discussion}

In the proceeding section, the results of the present study (viscosity evolution and structural rebuilding) will be tied in with those obtained in previous FBRM and formwork pressure studies that were performed by other researchers. The aim is to gain a better understanding of the effect of clays on the fresh state by observing clay-modified systems at multiple scales.

\subsection{FBRM and linear packing density model}

In a separate study, the microstructure of concentrated cementclay suspensions was directly examined through scanning laser microscopy, where an FBRM probe was used to measure the floc size evolution under different shear stresses [2]. Cement pastes with and without clay (1.5\% by mass of cement) were subjected to alternating cycles of low $(40 \mathrm{rpm})$, high $(400 \mathrm{rpm})$, and low (40 rpm) mixing intensities. The clay was sepiolite, a hydrated magnesium silicate. Sepiolite and palygorskite (the nanoclay used

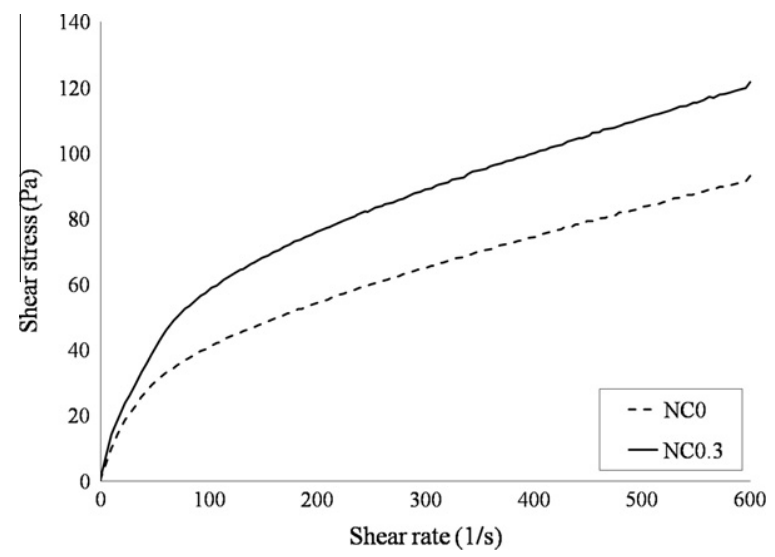

Fig. 11. Equilibrium flow curve obtained from SRE protocol.

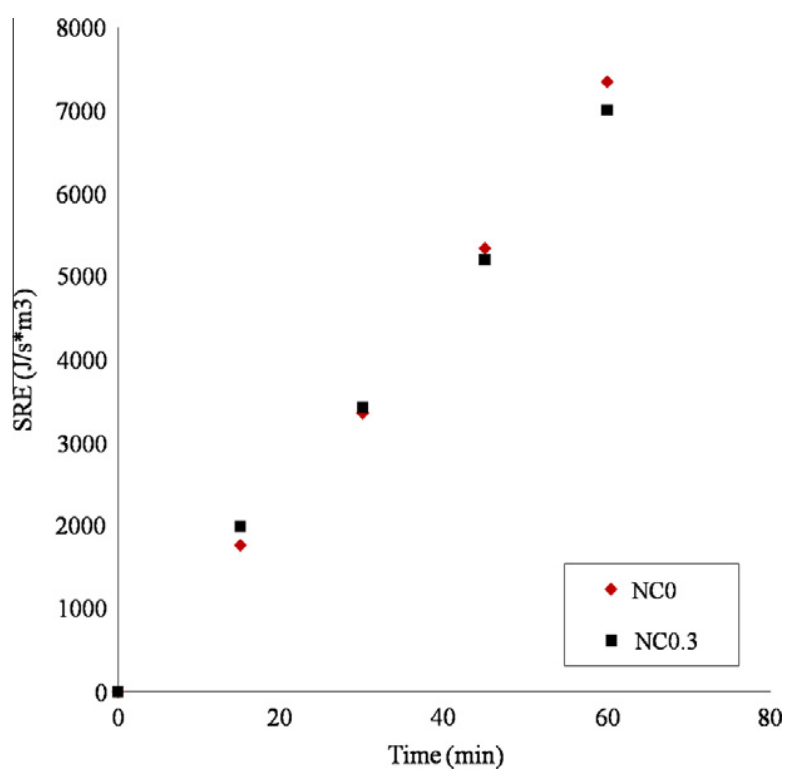

Fig. 12. SRE plot of pastes with and without a $0.3 \%$ nanoclay addition.

in the present study) are both phyllosilicates, and thereby share similar physical and physico-chemical properties [28]. The effect of sepiolite on the flocculation behavior of cement paste should reflect that of palygorskite.

The results are shown in Fig. 13, where floc size is given by average chord length. It is apparent that the clays increase floc size the cement paste with clay exhibited higher mean chord length throughout the cycles. However, during the low intensity cycles, both pastes experienced a similar evolution in chord length. Although the clay mix started off the cycle more flocculated at around $2500 \mathrm{~s}$, the rate of floc growth was similar to that of the plain cement paste. This suggests that the clays had an immediate effect on the degree of flocculation (upon introduction or removal of high shear) but had little influence over time, during which the rate of flocculation appears to be dominated by the cement phase. This was observed in the CR and SRE tests of the present study, as well.

Extending the linear packing density model to the results of the FBRM test, information about $\phi_{M}$ can be extracted. Upon removal of high shear, both pastes exhibited an immediate increase in average floc size. Therefore, it can be assumed that during the low

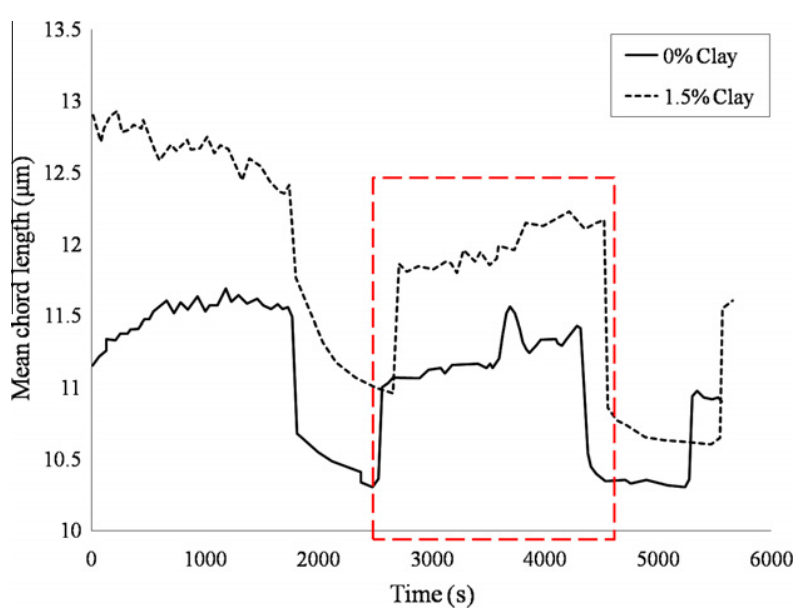

Fig. 13. FBRM results showing the evolution of mean chord length for pastes with and without a clay addition [2]. 
Table 5

Equilibrium flow curve parameters obtained from SRE protocol.

\begin{tabular}{lll}
\hline Mix & Viscosity (Pa-s) & Yield stress (Pa) \\
\hline NCO & 0.0958 & 35.7 \\
NC0.3 & 0.1104 & 55.3
\end{tabular}

shear cycle both pastes are in a highly flocculated state and will follow the $\phi_{M, 1}$ curve in Fig. 3. Over this cycle, the average floc size increases at a similar rate for both pastes. This translates to decreasing $\left[\phi_{2}\right]$ (implying increase of flocs) on the $\phi_{M, 1}$ curve, which corresponds to decreasing packing density. Referring back to the Krieger-Dougherty equation, decreasing $\phi_{M}$ will result in increasing viscosity, as was measured in the $\mathrm{CR}$ protocol after equilibrium.

\subsection{Application}

The results of the shear rheology tests were supplemented with those of the FBRM test, leading to a better understanding of how nanoclays affect flow behavior. The immediate increase in stiffness upon removal of shear is due to flocculation, an incremental jump in $\phi_{f}$ induced by the clays. With the reintroduction of shear, the material will experience a significant decrease in $\phi_{f}$ but continue to exhibit higher viscosity compared to neat paste due to increased flocculation strength and floc size, $\phi_{M, N C 1}<\phi_{M, N C O}$. The increase in stiffness over time (i.e. $\Delta \eta$ and $\Delta$ SRE), however, is similar for both pastes with and without clays. This may be attributed to decreasing $\phi_{M}$ governed by the cement particles. Here, this behavior will be correlated with the response of clay modified SCC mixes for formwork pressure.

\subsection{Formwork pressure}

As aforementioned, in a previous study the formwork pressure response of SCC with $0 \%(\mathrm{NCO})$ and $0.33 \%$ (NC0.33) clays were measured, results of which are shown in Fig. 2. The basic concrete mix proportions are given in Table 6. As shown in Fig. 2, the applied vertical pressure, $\sigma_{V}$, followed a step-wise loading protocol, where the load was increased incrementally every $.5 \mathrm{~h}$ and held. In an actual casting situation, $\sigma_{V}$ would increase gradually as SCC is being poured in the formwork before leveling off, as shown in Fig. 14, where the slope of $\sigma_{V}$ is representative of casting rate. A two-function model proposed by Kwon et al. [29] and Kim et al. [30] can describe the measured lateral pressure, $\sigma_{L}$, from parameters obtained through a step-wise loading protocol by introducing a loadingtime dependency. In this model, the pressure ratio between $\sigma_{V}$ and $\sigma_{L}$ is expressed by two functions as follows:

$\Delta \sigma_{L}\left(t, t^{\prime}\right)=\alpha\left(t, t^{\prime}\right) \beta\left(t^{\prime}\right) \Delta \sigma_{V}\left(t^{\prime}\right)$

where $\alpha$ is delayed response, $\beta$ is instantaneous response, $t$ is current time, and $t^{\prime}$ is time of loading. Further, while the concrete is plastic (before final set), $\alpha$ and $\beta$ can be represented in terms of delayed coefficient $a$ and instantaneous coefficient $b$, both of which are material parameters:

$\alpha\left(t, t^{\prime}\right)=1-a^{2} \cdot t^{\prime} \cdot\left(t-t^{\prime}\right)$

$\beta\left(t^{\prime}\right)=1-b \cdot t^{\prime}$

Table 6

The basic composition of SCC in formwork pressure study expressed in $\mathrm{kg}$ to produce $1 \mathrm{~m}^{3}$ of concrete.

\begin{tabular}{llllll}
\hline Ingredient & Water & Cement & Fly ash & Sand & Gravel \\
\hline Amount & 182 & 338 & 154 & 879 & 824 \\
\hline
\end{tabular}

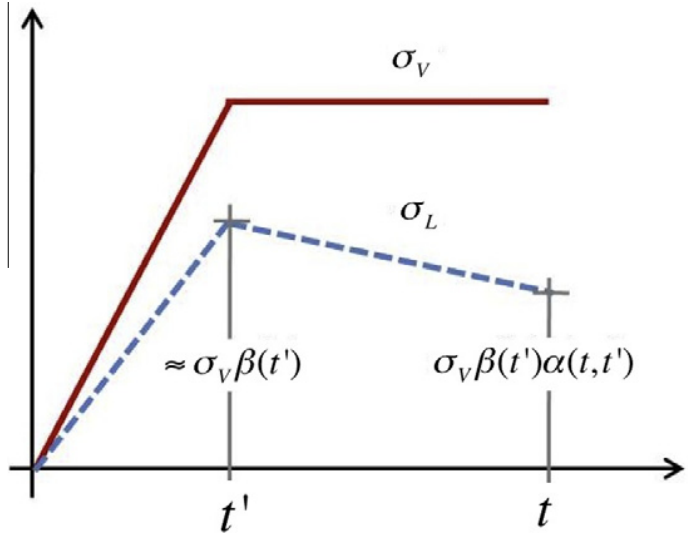

Fig. 14. Two function model for formwork pressure of concrete [30].

In the CR and SRE protocols, an equilibrium state was established for a given constant shear rate, which provided a well-defined initial reference condition. In the loading protocol followed in the formwork pressure study, each step-wise increase in vertical pressure is an increase in volumetric strain. At each applied constant volumetric strain rate, a new equilibrium state is established. (It is assumed that the material can reach equilibrium very rapidly due to the high applied load.) This allows for the formwork pressure response to be described through $\phi_{M}$.

The delayed and instantaneous response of NCO and NC0.33 are shown in Fig. 15a and b, respectively. Upon increase in volumetric strain, NC0.33 experienced lower lateral pressure compared to NCO, as shown in the evolution of $\beta$ in Fig. 15b. The $\beta\left(t^{\prime}\right)$ curve for $\mathrm{NC} 0.33$ is steeper, indicating $\mathrm{NCO} .33$ experienced increasingly reduced $\sigma_{L}$ at each incremental increase in $\sigma_{V}$ compared to NCO. This can be attributed to the lower $\phi_{M}$ induced by the nanoclays. Similarly, a lower $\phi_{M}$ led to higher $\eta$ at equilibrium in the CR protocol and higher yield stress and dynamic viscosity at equilibrium in the SRE protocol. As the volumetric strain was held constant over $.5 \mathrm{~h}$, lateral pressure decreased at a similar rate for both SCC mixes, as shown in the evolution of $d \alpha\left(t, t^{\prime}\right)$ / $d\left(t-t^{\prime}\right)$ in Fig. 15a. This occurred because the rate at which $\phi_{M}$ decreased under constant loading was governed by the properties of the cement and fly ash particles, not the nanoclays. This correlates well with the flow behavior measured in this study, where clays had an immediate effect but have little or no influence over time.

For the design of formwork, the maximum formwork pressure, $\sigma_{\text {max }}$, is a critical value. The maximum formwork pressure depends only on placement rate and material properties (i.e. unit weight of concrete and coefficients $a$ and $b$ ), not time. In a formwork pressure study by Kim et al. [30], which used the two-function model to characterize a variety of different SCC mixes, a much greater variation was found for coefficient $b$ than $a$. Also, the variation in a had a negligible effect on $\sigma_{\max }$ while the variation in b had a dominant one during the first few hours of casting. It is apparent from Fig. 15b that the instantaneous increase in $\phi_{M}$ induced by the nanoclays significantly affects coefficient $b$. It follows that nanoclays have the capacity to greatly reduce $\sigma_{\max }$, which explains why they are effective in modifying SCC for the application of reduced formwork pressure.

In the present study, the influence of clays on the flow behavior of systems with cement was investigated. Since SCC systematically incorporates fly ash, viscosity modifying agents and superplasticizers, the interaction of clays with these constituents and the effect on rheology must be explored, as well. Work is currently being done and will be reported in the future. 

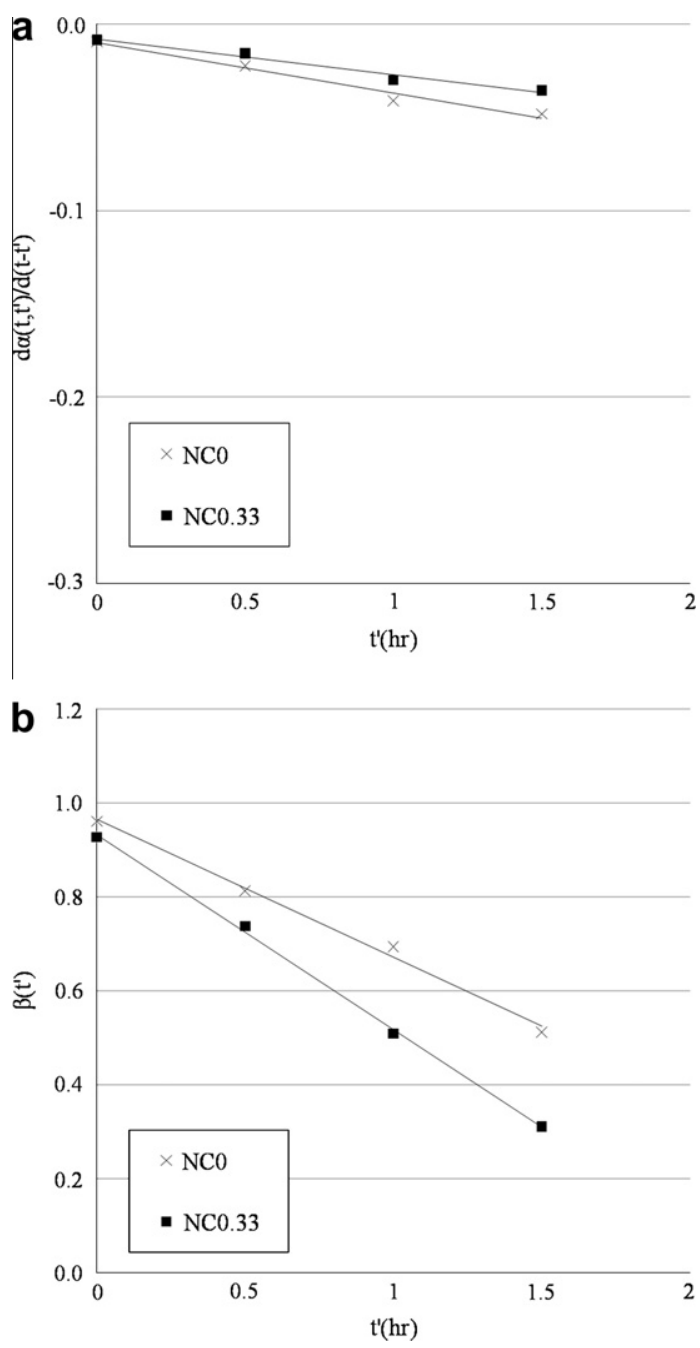

Fig. 15. (a) Delayed response and (b) instantaneous response.

\section{Conclusions}

Examining concentrated cement-clay systems at multiple scales gave insight into how the clays affect fresh-state properties. The effective solid volume fraction and maximum packing density were useful parameters in describing how the clays influence the macroscale flow properties of pastes over time. It is apparent that the clays have an immediate effect on stiffness, and the governing mechanism is flocculation, not water adsorption leading to loss of free water. However, under a constant shear rate, the clays have little or no influence over time - stiffening is dominated by changes in $\phi_{M}$ caused by the powder particles such as cement and/or fly ash. The parameters $\phi_{f}$ and $\phi_{M}$ were ultimately used to explain the response of SCC concrete for lab-scale simulations of formwork pressure, from which it was apparent why clays are an effective mineral admixture in modifying thixotropy of SCC for this application.

\section{Acknowledgements}

The authors would like to acknowledge the financial support from the Infrastructure Technology Institute at Northwestern
University and Tennessee Valley Authority (TVA) and Oak Ridge Associated Universities (ORAU) (Award 105866); and Active Minerals, WR Grace and Lafarge for providing materials.

\section{References}

[1] Tregger NA, Pakula ME, Shah SP. Influence of clays on the rheology of cement pastes. Cem Concr Res 2010;40(Compendex):384-91.

[2] Ferron R. Formwork pressure of self-consolidating concrete: influence of flocculation mechanisms, structural rebuilding, thixotropy and rheology. PhD Thesis, Northwestern University, Evanston, IL; 2008.

[3] Kuder KG, Shah SP. Rheology of extruded cement-based materials. ACI Mater J 2007;104(3):283-90.

[4] Pekmezci BY, Voigt T, Kejin W, Shah SP. Low compaction energy concrete for improved slipform casting of concrete pavements. ACI Mate J 2007;104 (Compendex):251-8.

[5] Tregger N, Voigt T, Shah S. Improving the slipform process via material manipulation. Adv Constr Mater 2007:539-46 [Grosse CU, Springer Berlin Heidelberg].

[6] Tregger N. Tailoring the fresh state of concrete. PhD Thesis, Northwestern University, Evanston, IL; 2010.

[7] Voigt T, Mbele J-J, Wang K, Shah SP. Using fly ash, clay, and fibers for simultaneous improvement of concrete green strength and consolidatability for slip-form pavement. J Mater Civ Eng 2010;22(2):196-206.

[8] Kim JH, Beacraft M, Shah SP. Effect of mineral admixtures on formwork pressure of self-consolidating concrete. Cem Concr Compos 2010;32(9):665-71.

[9] Amziane S. Setting time determination of cementitious materials based on measurements of the hydraulic pressure variations. Cem Concr Res 2006;36(2):295-304.

[10] Senff L, Labrincha JA, Ferreira VM, Hotza D, Repette WL. Effect of nano-silica on rheology and fresh properties of cement pastes and mortars. Constr Build Mater 2009;23(7):2487-91.

[11] Barnes HA. Thixotropy - a review. J Nonnewton Fluid Mech 1997;70 $(1-2): 1-33$.

[12] Assaad J, Khayat $\mathrm{KH}$, Mesbah $\mathrm{H}$. Variation of formwork pressure with thixotropy of self-consolidating concrete. ACI Mater J 2003;100(1):29-37.

[13] Ovarlez G, Roussel N. A physical model for the prediction of lateral stress exerted by self-compacting concrete on formwork. Mater Struct 2006;39(2):269-79.

[14] Krieger IM, Dougherty TJ. A mechanism for non-newtonian flow in suspensions of rigid spheres. Trans Soc Rheol 1959;3(1):137-52.

[15] Krieger IM. Rheology of monodisperse latices. Adv Colloid Interface Sci 1972;3(2):111-36.

[16] Ball RC, Richmond P. Dynamics of colloidal dispersions. Phys Chem Liq: Int J 1980;9(2):99-116

[17] Soua Z, Larue O, Vorobiev E, Lanoisellé J-L. Estimation of floc size in highly concentrated calcium carbonate suspension obtained by filtration with dispersant. Colloids Surf, A 2006;274(1-3):1-10.

[18] Struble L, Sun G-K. Viscosity of Portland cement paste as a function of concentration. Adv Cem Based Mater 1995;2(2):62-9.

[19] Mansoutre S, Colombet P, Van Damme H. Water retention and granular rheological behavior of fresh C3S paste as a function of concentration. Cem Concr Res 1999;29(9):1441-53.

[20] Tregger N, Knai H, Shah SP. Flocculation behavior of cement pastes containing clays and fly ash. In: Transition from Fluid to Solid: Re-examining the Behavior of Concrete at Early Ages - Technical Session at the 2009 ACI Spring Conference. American Concrete Institute; March 15, 2009-March 19, 2009. p. $127-38$

[21] Stovall T, de Larrard F, Buil M. Linear packing density model of grain mixtures. Powder Technol 1986;48(1):1-12.

[22] Ferron R, Shah S. The fresh state. Transport Res Rec: J Transport Res Board 2010;2141(-1):89-91.

[23] Active Minerals Company LLC, What is Acti-Gel 208 and how is it made? 2007

[24] Galán E, Mesa J, Sánchez C. Properties and applications of palygorskite clays from ciudad real, central spain. Appl Clay Sci 1994;9(4):293-302.

[25] ASTM C 1611/C 1611M-09b. Slump flow of self-consolidating concrete, ASTM International, West Conshohocken, PA; 2009.

[26] Ferron R, Gregori A, Sun Z, Shah SP. Rheological method to evaluate structural buildup in self-consolidating concrete cement pastes. ACI Mater J 2007;104(3):242-50.

[27] Papanastasiou TC. Flows of materials with yield. J Rheol 1987;31(5):385-404

[28] Galan E. Properties and applications of palygorskite-sepiolite clays. Clay Miner 1996;31(4):443-53.

[29] Kwon SH, Shah SP, Phung QT, Kim JH, Lee Y. Intrinsic model to predict formwork pressure. ACI Mater J 2010;107(1):20-6.

[30] Kim JH, Beacraft M, Kwon SH, Shah SP. Simple analytical model for formwork design of self-consolidating concrete. ACI Mater J 2011;108(1):38-45.

[31] Mbele J-J. Optimization of self-consolidating concrete for slip-form pavement. MS Thesis, Northwestern University, Evanston, IL; 2006. 